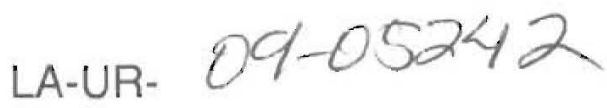

Approved for public release

distribution is unlimited.

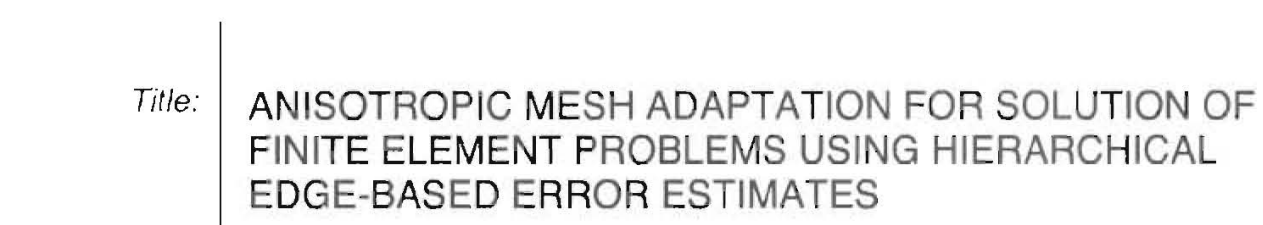

Author(s): $\quad$ KONSTANTIN LIPNIKOV

ABDELLATIF AGOUZAL

YURI VASSILEVSKI

Intended for:

PROCEEDINGS:

18TH INTERNATIONAL MESHING ROUNDTABLE



Los Alamos National Laboratory, an affirmative action/equal opportunity employer, is operated by the Los Alamos National Security, LLC for the National Nuclear Security Administration of the U.S. Department of Energy under contract DE-AC52-06NA25396. By acceptance of this article, the publisher recognizes that the U.S. Government retains a nonexclusive, royalty-free license to publish or reproduce the published form of this contribution, or to allow others 10 do so, for U.S. Government purposes. Los Alamos National Laboratory requests that the publisher identify this article as work performed under the auspices of the U.S. Department of Energy. Los Alamos National Laboratory strongly supports academic freedom and a researcher's right to publish; as an institution, however, the Laboratory does not endorse the viewpoint ol a publication or guarantee its technical correctness.

Form $836(7 / 06)$ 



\title{
Anisotropic mesh adaptation for solution of finite element problems using hierarchical edge-based error estimates
}

\author{
Abdellatif Agouzal' ${ }^{1}$ Konstantin Lipnikov², and Yuri Vassilevski.3 \\ 1 Universite de Lyon 1, Laboratoire d'Analyse Numerique, agouzalouniv-lyon1.fr \\ 2 Los Alamos National Laboratory, Theoretical Division, lipnikov@lanl.gov \\ 3 Institute of Numerical Mathematics, vasilevs@dodo.inm.ras .ru
}

Summary. We present a new technology for generating meshes minimizing the inlerpolation and discretization errors or their gradients. The key element of this methodology is construction of a space metric from edge-based error estimates. For a mesh with $N_{h}$ triangles, the error is proportional to $N_{h}^{-1}$ and the gradient of crror is propoltional to $N_{h}^{-1 / 2}$ which are optimal asymptotics. The methodology is verified with numerical experiments.

\section{Introduction}

Unstructured simplicial meshes are ideally suited for adaptive finite element calculations. The simplexes can be aligned with solution features and cover the computational domain in an optimal way to equiclistribute the error. This results in a smaller computational mesh and potentially faster calculations.

Generation of optimal adaptive meshes requires error estimates or error indicators that carry directional information about the solution. In this article, we use elror estimates that are associated with mesh edges. We consider edge-based error estimates for the interpolation error and hierarchical error estimates for the discretization error $[10]$. In both cases, we employ the methodology developed in $[1,3.4]$ for the interpolation error. This methodology results in a metric that captures correctly isotropic and anisotropic solution features. Here, we continue analysis of this metric, in particular, its smoothness and anisotropic alignment.

Other methods for generating a space metric are often based on the Hessian of the discrete solution $[16,13,12,11]$. For such a metric, optimal error estimates for the interpolation error have been proved in $[2,6,12,15.16]$. The Hessian-based netric has been successfully applied to adaptive solution of PDEs [5, 11, 13]. However, its theoretical analysis requires to make additional assumptions that the discrete Hessian approximates the continuous 
one in the maximum norm. Despite the fact that this assumption is frequently violated in many Hessian recovery methods, the generated adaptive meshes still result in optimal error reduction.

The technology, which we proposed in $[1,3,4]$ removes problems associated with the Hessian recovery methods. It can be also applied to adaptive solution of finite element problems. The price to pay is that error estimates or error indicators have to be prescribed to mesh edges. The finite element literature provides a number of ways to obtain these error estinates. In this article, we use the hierarchical crror estimates from [10] and provide a numorical analysis of our methodology for the adaptive solution of finite elenent problems. The cornerstone of this methodology is construction of a space metric from edgebased error estimates.

We define a tensor metric $\mathfrak{M}$ such that the volume and the perimeter of a simplex measured in this metric control the norn of error or its gradient. The equidistribution principle, which can be traced lrack to D'Azevedo [9],

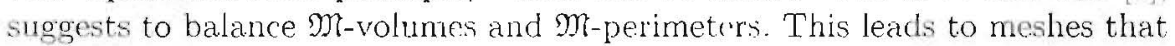
are quasi-uniform in the piecewise constant metric $\mathfrak{M}$. The piecewise constant metric may produce instabilities in an adaptivc process, especially when the length of a mesh edge, measured in all metrics asnociated with simplexes sharing the edge, varies strongly. We show numerically that this variation is relatively small for our piecewise constant metrics. This allows us to convert. the piecewise constant metric into a continuous one for additional robustness of the adaptive process.

The paper outline is as follows. In Section 2, we derive metrics optimal for the interpolation errors. In Section 3, we present the algorithm for generating adaptive meshes. in Section 4 , we apply the methodology for adaptive solution of finite element problems.

Acknowledgments. Research of the third author has been supported partially by the RFBR project 08-01-00159-a.

\section{Interpolation error analysis}

\subsection{Edge-based error estimates and a tensor metric}

Let $\Omega \subset \Re^{d}$ be a bounded polyhedral domain and $\Omega_{h}$ be a conformal simplicial mesh with $N_{h}$ simplexes. Let $\mathfrak{M}$ be a piecewise constant tensor metric on $\Omega_{h}$. The volume of simplex $\Delta$ and the length of edge $e$ in this metric are denoted by $|\Delta|_{\mathfrak{m}}$ and $|\mathbf{e}|_{\mathfrak{m}}$, respectively [2]. The total length of all edges of simplex $\Delta$ in denoted by $\left.\partial \Delta\right|_{\mathfrak{M}}$. We shall refer to $|\partial \Delta|_{\mathfrak{M}}$ as the perimeter of $\Delta$ in the metric $\mathfrak{M}$.

Let $\mathcal{I}_{1} u$ be the piecewise linear Lagrange interpolant of $u$, and $\mathcal{I}_{1, \Delta u} u$ be its restriction to $\Delta$. Similarly, let $\mathcal{I}_{2} u$ be the piecewise quadratic Lagrange interpolant of $u$, and $\mathcal{I}_{2, \Delta} u$ be its restriction to $\Delta$. Our goal is to generate a mesh 
that minimizes (approximately) the $L^{p}$-norm, $p \in(0, \infty)$, of the interpolation error

$$
e=u-\mathcal{I}_{1} u
$$

or its gradicnt $\nabla e$. A sequence of meshes with increasing number of simplexes must provide the optinal reduction of this error. For instance, the Tichomirov result $[14]$ inplies that the optimal reduction of the $L^{\infty}$-norm of error is proportional to $N_{h}^{-1}$.

Let us consider a particular $d$-simplex $\Delta$ with vertices $v_{i}, i=1, \ldots, d+1$, edge vectors $\mathrm{e}_{k}=\mathrm{v}_{i}-\mathrm{v}_{j}, \mathrm{I} \leq i<j \leq d+1$, and mid-edge points $\mathrm{c}_{k}$, $k=1, \ldots, n_{d}$, where $n_{d}=d(d+1) / 2$. Let $\lambda_{i}, i=1, \ldots, d+1$, be the linear functions on $\Delta$ such that $\lambda_{i}\left(v_{j}\right)=\delta_{i . j}$ where $\delta_{i j}$ is the Kronecker symbol. For every edge $e_{k}$, we define the quadratic bubble function $b_{k}=\lambda_{i} \lambda_{j}$.

Let $u$ be a continuous function and $u_{2}=\mathcal{I}_{2, \Delta} u$ be its quadratic approximation on $\Delta$. We have

$$
e_{2}=u_{2}-\mathcal{I}_{1 . \Delta u_{2}}=4 \sum_{k=1}^{n_{\mu}}\left(u_{2}\left(c_{k}\right)-\mathcal{I}_{1, \Delta} u_{2}\left(\mathrm{c}_{k}\right)\right) b_{k} \equiv \sum_{k=1}^{n_{u}} \gamma_{k} b_{k} .
$$

The $L^{2}$-norm of the error $e_{2}$ is given by

$$
\left\|e_{2}\right\|_{L^{2}(\Delta)}^{2}=|\Delta|(\mathbb{B} \gamma, \gamma)
$$

where $\gamma$ is a vector with $n_{d}$ components $\gamma_{k}$ and $\mathbb{B}$ is the $n_{d} \times n_{d}$ symmetric positive definite scaled Gramm matrix with positive entries $\mathbb{B}_{k, l}=$ $|\Delta|^{-1} \int_{\Delta} b_{k} \cdot b_{l} \mathrm{~d} V$. Note that (2) is only a number; therefore, it does not provide any directional information. To recoven this information, we split the error into $n_{d}$ pieces associated with edges of $\Delta$ :

$$
\left\|e_{2}\right\|_{L^{2}(\Delta)}=|\Delta|^{1 / 2} \sum_{k=1}^{i i_{d}} \alpha_{k} \quad \text { and } \quad \sum_{k=1}^{n_{d}} \alpha_{k}=(\mathbb{B} \gamma, \gamma)^{1 / 2} \text {. }
$$

Not careful selection of $\alpha_{k}$ may result in loss of directional information. In the sequel, we motivate the following choice of $\alpha_{k}$ :

$$
\alpha_{k}=\left|\gamma_{k}\right|(\mathbb{B} \gamma, \gamma)^{1 / 2}\left(\sum_{k=1}^{n_{d}}\left|\gamma_{k}\right|\right)^{-1}
$$

We repeat the above derivations for the gradient of the error. The $L^{2}$-norm of $\nabla e_{2}$ is given by

$$
\left\|\nabla e_{2}\right\|_{L^{2}(\Delta)}^{2}=\left\|\sum_{k=1}^{n_{d}} \gamma_{k} \nabla b_{k}\right\|_{L^{2}(\Delta)}^{2}=|\Delta|(\widetilde{\mathbb{B}} \gamma, \gamma)
$$

where $\widetilde{\mathbb{B}}$ is the $n_{d} \times n_{d}$ symmetric positive definite matrix with entries $\widetilde{\mathbb{B}}_{k, l}=$ $|\Delta|^{-1} \int_{\Delta} \nabla b_{k} \cdot \nabla b_{l} d V$. Again, we split this error (a lumber) into $n_{d}$ edge-based error estimates $\tilde{\alpha}_{k} \geq 0$ sur h that 


$$
\left\|\nabla c_{2}\right\|_{L^{2}(\Delta)}^{2}=|\Delta| \sum_{k=1}^{n_{l l}} \tilde{\alpha}_{k} \quad \text { and } \quad \sum_{k=1}^{n_{d} l} \tilde{\alpha}_{k}=(\widetilde{\mathbb{B}} \gamma, \gamma) .
$$

Again, a proper choice of $\alpha_{k}$ is required to preserve directional information in a final metric. In the seguel, we motivate the following choice of $\tilde{\alpha}_{k}$ :

$$
\bar{\alpha}_{k}=\left|\gamma_{k}\right|(\widetilde{\mathbb{B}} \gamma, \gamma)\left(\sum_{k=1}^{n_{k}}\left|\gamma_{k}\right|\right)^{-1}
$$

In both cases, the edges-based errors $a_{k}$ and $\tilde{a}_{k}$ are proportional to $\left|\gamma_{k}\right|$ which is the same for all simplexes sharing the edge $\boldsymbol{e}_{k}$. This observation is a key to understanding 'smoothness' of the metric whose element-by-element construction is based on the following result $[1,4]$.

Lemma 1. Let $\alpha_{k}, k=1, \ldots, n_{d}$, be values prescribed to edges of a d-simplex $\triangle$ such that

$$
\alpha_{k} \geq 0 \quad \text { and } \quad \sum_{k=1}^{n_{d}} \alpha_{k}>0
$$

Then, there exists a constant tensor metric $\mathfrak{M}_{\Delta}$ such that

$$
\left(\frac{d !}{(d+1)(d+2)}\right)^{1 / d}|\Delta|_{m_{\Delta}}^{2 / d} \leq \sum_{k=1}^{n_{n}} \alpha_{k} \leq|\partial \Delta|_{\eta_{\Delta}}^{2} .
$$

The proof $[1,4]$ of Lemma 1 provides the constructive way to define the metric $\mathfrak{M}_{\Delta}$. Due to its importance, we present a shortened proof here

Proof. Let us define the quadratic function

$$
v_{2}=-\frac{1}{2} \sum_{k=1}^{n_{d}} c k_{k} b_{k} .
$$

The trace of $v_{2}$ on $e_{k}$ is a quadratic function $w_{2}$ vanishing at endpoints $\mathbf{v}_{i}, \mathbf{v}_{j}$ of $\mathrm{e}_{k}$ with an extremum at $\mathrm{c}_{k}$. Therefore, $w_{2}^{\prime}\left(\mathrm{c}_{k}\right)=0$ and $\nabla v_{2}\left(\mathrm{c}_{k}\right) \cdot \mathrm{e}_{k}=0$. Let $\mathbb{H}$ be the Hessian of $v_{2}$. Apptying the multi-point Taylor formula $[7,8]$ for $v_{2}$ at endpoints $\mathbf{v}_{i}$ and $\mathbf{v}_{j}$ of $\mathbf{e}_{k}$, we get

$$
\begin{aligned}
& 0=v_{2}\left(\mathbf{v}_{i}\right)=v_{2}\left(\mathbf{c}_{k}\right)-\frac{1}{2} \nabla v_{2}\left(\mathbf{c}_{k}\right) \cdot \mathbf{e}_{k}+\frac{1}{8}\left(\mathbb{H} \mathbf{e}_{k}, \mathbf{e}_{k}\right), \\
& 0=v_{2}\left(\mathbf{v}_{j}\right)=v_{2}\left(\mathbf{c}_{k}\right)+\frac{1}{2} \nabla v_{2}\left(\mathbf{c}_{k}\right) \cdot \mathbf{e}_{k}+\frac{1}{8}\left(\mathbb{H} \mathbf{e}_{k}, \mathbf{e}_{k}\right) .
\end{aligned}
$$

Thus:

$$
\left(\mathbb{H} \mathbf{e}_{k}, \mathbf{e}_{h}\right)=\alpha_{k} .
$$

The Hessian $\mathbb{H}$ may be indefinite and hence cannot be used to define the metric $\mathfrak{M}_{\Delta}$. In order to make it positive semidefinite, we take its spectral module: 


$$
|\mathbb{H}|=W^{T}|\Lambda| W
$$

where $\mathbb{H I}=W^{T} \Lambda W$ is the spectral decomposition of the symmetric matrix $\mathbb{H}$.

If $\operatorname{det} \mathbb{H} \neq 0$, we set $\mathfrak{M}_{\Delta}=|\mathbb{H}|$. The upper bound follow's from

$$
\left|\partial \Delta_{|\mathbb{H}|}^{2}=\left(\sum_{k=1}^{n_{d}}\left(|\mathbb{H}| \mathbf{e}_{k}, \mathbf{e}_{k}\right)^{1 / 2}\right)^{2} \geq \sum_{k=1}^{n_{d}}\left(\| \mathbb{H} \mid \mathbf{e}_{k}, \mathbf{e}_{k}\right) \geq \sum_{k=1}^{n_{k}}\right|\left(\mathbb{H} \mid \mathbf{e}_{k}, \mathbf{e}_{k}\right) \mid=\sum_{k=1}^{n_{d}} a_{k} \cdot
$$

To estimate the lower bound, we use formula for the Cayley-Menger doterminant generalized to the case $\mathbb{H} \mathbb{I} \neq \mathbb{I}$ (for its proof we refer to [1]):

$$
\operatorname{det}(\mathbb{H})|\Delta|^{2}=\frac{(-1)^{d-1}}{2^{d}(d !)^{2}} \operatorname{det}(K(\mathbb{H}))
$$

wherc

$$
K(\mathbb{H})=\left(\begin{array}{cccc}
\left(\mathbb{H} \mathbf{v}_{11}, \mathbf{v}_{11}\right) & \cdots & \left(\mathbb{H} \mathbf{v}_{l d_{1}}, v_{1 d_{1}}\right) & 1 \\
\vdots & \ddots & \vdots & \vdots \\
\left(\mathbb{H} v_{d_{1} 1}, \mathbf{v}_{d_{1} 1}\right) & \cdots & \left(\mathbb{H} v_{d_{1} d_{1}}, v_{d_{1} d_{1}}\right) & 1 \\
1 & \cdots & 1 & 0
\end{array}\right)
$$

and $\mathbf{v}_{i j} \equiv \mathbf{v}_{i}-\mathbf{v}_{j}$. Therefore,

$$
\begin{aligned}
|\Delta|_{|| \mathbb{H} \mid}^{2}=\operatorname{det}(|\mathbb{H}|)|\Delta|^{2} & =\frac{(-1)^{d-1}}{2^{d}(d !)^{2}} \operatorname{det}(K(|\mathbb{H}|)) \\
& \leq \frac{1}{2^{d}(d !)^{2}} \sup _{\alpha \in \mathbb{R}^{n_{d}}} \frac{\operatorname{det} K(\mathbb{H}) \mid}{\max _{1 \leq k \leq n_{d}} \alpha_{k}^{d}}\left(\sum_{k=1}^{n_{d}} \alpha_{k}\right)^{d} .
\end{aligned}
$$

For a square matrix $K(\mathbb{H})$ with elements $k_{i, j}$, it holds

$$
|\operatorname{det}(K(\mathbb{H}))| \leq\left|\sum_{\sigma} \prod_{i=1}^{d+2} k_{i, \sigma_{i}}\right| \leq(d+2) ! \max _{\sigma}\left|\prod_{i=1}^{d+2} k_{i, \sigma_{,}}\right|,
$$

where the summation is performed over all possible permutations $\sigma$ of matrix rows and columns. Since $k_{i, j}=\left(\mathbb{H} e_{k}, e_{k}\right)=\alpha_{k}, 1 \leq i<j \leq d_{1}$, from (10) we derive that $\operatorname{det}(K(\mathbb{H}))$ is a homogeneous polynomial of degree $d$ of $\alpha_{k}$ and

$$
\sup _{\alpha \in \Re^{n_{d}}} \frac{|\operatorname{det} K(\mathbb{H})|}{\max _{1 \leq k \leq n_{d}} \alpha_{k}^{d}} \leq(d+2) ! \sup _{\alpha \in \Re^{n_{d}}} \frac{\max _{1 \leq k \leq n, i} \alpha_{k}^{d}}{\max _{1 \leq k \leq n d} \alpha_{k}^{d}} \leq(d+2) ! .
$$

Therefore, we conclude from (11) that

$$
|\Delta|_{|H|}^{2} \leq \frac{1}{2^{d}} \frac{(d+1)(d+2)}{d !}\left(\sum_{k=1}^{n_{l}} \alpha_{k}\right)^{d}
$$

which implies the lower bound in (7). 
If $\operatorname{det}(\mathbb{H})=0$, the Hessian $\mathbb{H}$ cannot be used to generate a metric. In this case, we modify $\alpha_{k}$ to get a new quadratic function $v_{2}$ with a non-degenerate Hessian such that (7) is still satisfied. For the sake of simplicity, we restrict ourselves to the case $0 \leq \alpha_{1} \leq \alpha_{2} \leq \cdots \leq \alpha_{n_{d}}$ and $\alpha_{n_{d}} \neq 0$. The modified edge data are

$$
\bar{\alpha}_{k}=\alpha_{k}, \quad k=1, \ldots, n_{d}-1, \quad \tilde{\alpha}_{n_{d}}=(1+\delta) \alpha_{n_{d}} .
$$

where $\delta \in] 0,1]$.

Let $\bar{\tau}_{2}(\delta)=-\frac{1}{2} \sum_{k=1}^{n_{d}} \tilde{\alpha}_{k} b_{k}$ be the modified quadratic function and $\tilde{\mathbb{H}}(\delta)$ be its Hessian. Formulas (9) and (10) imply that $p(\delta) \equiv \operatorname{det}(\widetilde{\mathbb{H}}(\delta))$ is a polynomial of degree two. Since $p(0)=\operatorname{det}(\mathbb{H})=0$, there exists $\delta_{0} \in[0,1]$ such that $\operatorname{det}\left(\tilde{\mathbb{H}}\left(\delta_{0}\right)\right) \neq 0$. We set $\mathfrak{M}_{\Delta}=\left|\widetilde{\mathbb{H}}\left(\delta_{0}\right)\right|$ and check that

$$
\sum_{k=1}^{n_{d} d} \alpha_{k} \leq \sum_{k=1}^{n_{d}} \tilde{\alpha}_{k} \leq \sum_{k=1}^{n_{d}}\left(\left|\tilde{\mathbb{H}}\left(\delta_{0}\right)\right| \mathbf{e}_{k}, \mathbf{e}_{k}\right) \leq\left(\sum_{k=1}^{n_{d}}\left(\left|\tilde{\mathbb{H}}\left(\delta_{0}\right)\right| \mathbf{e}_{k}, \mathbf{e}_{k}\right)^{1 / 2}\right)^{2}=|\partial \Delta|_{\Omega_{N}}^{2} \Delta
$$

and

$$
\sum_{k=1}^{n_{k}} \alpha_{k} \geq \frac{1}{2} \sum_{k=1}^{n_{i}} \tilde{\alpha}_{k} \geq\left(\frac{(d+1)(d+2)}{d !}\right)^{-\frac{1}{d}}|\Delta|_{\mathfrak{m}_{\lrcorner}}^{\frac{2}{2}} .
$$

This proves the assertion of the lomma.

Using Lemma 1 and norm definition (3), we build the auxiliary metric $\mathfrak{M}_{\Delta}$ for error $e_{2}$. Similarly; using Lemma $I$ and norm definition (5), we build the auxiliary metric $\overline{\mathfrak{M}}_{\Delta}$ for $\nabla e_{2}$. These metrics do not provide a geometric representation of the error since the error estimates involve also the volume of simplex in the Cartesian metric. This mismatch is fixed in the following section.

\subsection{Metrics for the $L^{p}$-norm of error and its gradient}

In this section, we consider $L^{p}$-norms, $p \geq 1$, of the errors as well as $I^{p}$-quasinorms, $0<p<1$. As described in [16, 1, 4], as well as in [12], the metrics controlling various $L^{p}$-norms differ by a scaling factor. Let

$$
\mathfrak{M}_{\Delta, p}=\left(\operatorname{det}\left(\mathfrak{M}_{\Delta}\right)\right)^{-1 /(d+2 p)} \mathfrak{M}_{\Delta} \quad \text { and } \quad \widetilde{\mathfrak{M}}_{\Delta, p}=\left(\operatorname{det}\left(\widetilde{\mathfrak{M}}_{\Delta}\right)\right)^{-1 /(d+p)} \widetilde{\mathfrak{M}}_{\Delta} \text {. }
$$

The following estimates are proved in $[16,1,4]$.

Lemma 2. Let $\mathfrak{M}_{\Delta, p}$ and $\widetilde{\mathfrak{M}}_{\Delta, p}$ be the constant tensor metrics defined above. Then,

$$
c_{p}|\Delta|_{\mathfrak{M}_{\Delta, p}}^{2 j d+1 / p} \leq\left\|e_{2}\right\|_{L^{\prime \prime}(\Delta)} \leq C_{p}|\Delta|_{\mathfrak{M}_{\Delta, n}}^{1 / p}|\partial \Delta|_{\mathfrak{M}_{\Delta, p}}^{2}
$$

and 


$$
\tilde{C}_{p}|\Delta|_{\widetilde{\mathfrak{M}}_{\Delta, p}}^{1 / d+1 / p} \leq\left.|| \nabla e_{2}\right|_{L^{\prime \prime}(\Delta)} \leq \tilde{C}_{p}|\Delta|_{\widetilde{\mathfrak{M}}_{\lrcorner, p}}^{1 / p}|\partial \Delta|_{\widetilde{\mathfrak{M}}_{\perp, p}},
$$

where constants $c_{p}, C_{p}, \tilde{c}_{p}, \tilde{C}_{p}$ depend only on $d$ and $p$.

For brevity, we confine ourselves to the case $p=\infty$. In this case, the constants in Lemma 2 depend only on $d$. Moreover, the metrics generated by Lemma $I$ are optimal, i.e. $\mathfrak{M}_{\Delta, \infty}=\mathfrak{M}_{\Delta}$ and $\widehat{\mathfrak{M}}_{\Delta . \infty}=\widetilde{\mathfrak{M}}_{\Delta}$.

\subsection{Extension to general functions}

For a given continuous function $u$, we use the computable error $e_{2}$ to estimate the true error $e$ :

$$
e=u-\mathcal{I}_{1, \Delta u}
$$

Let $\mathcal{F}$ be the space of symmetric $d \times d$ matrices and $|\mathbb{H}|$ be the spectral module of $\mathbb{H} \in \mathcal{F}$. We introduce the following notations:

$$
\left\|\mathrm{e}_{k}\right\| \|_{|\mathbb{H}|}^{2}=\max _{\mathrm{x} \in \Delta}\left(|\mathbb{H}(\mathrm{x})| \mathrm{e}_{k}, \mathrm{e}_{k}\right) \quad \text { and } \quad\|\partial \Delta\|_{j \mathbb{H} \mid}^{2}=\left.\sum_{k=1}^{n_{t i}}\left\|\mathbf{e}_{k}\right\|\right|_{|\mathbb{H}|} ^{2} .
$$

The following result is proved in $[1,4]$.

Lemma 3. Let $u \in C^{2}(\bar{\Delta})$. Then,

$$
\frac{d+1}{2 d}\left\|e_{2}\right\|_{L^{x}(\Delta)} \leq\|\|_{L \times(\Delta)} \leq\left\|e_{2}\right\|_{L \times(\Delta)}+\frac{1}{4} \inf _{\mathbb{F} \in \mathcal{F}}\|\partial \Delta\|_{|\mathbb{H}-\mathbb{F}|}^{2} .
$$

and

$$
\left\|\nabla e_{2}\right\|_{L^{\infty}(\Delta)}-\operatorname{osc}(\mathbb{H}, \Delta) \leq\|\nabla e\|_{L^{x}(\Delta)} \leq\left\|\nabla e_{2}\right\|_{L^{\infty}(\Delta)}+\operatorname{osc}(\mathbb{H}, \Delta),
$$

where the oscillation term is

$$
\operatorname{osc}(\mathbb{H}, \Delta)=C_{o s c} \frac{|\partial \Delta|^{d-1}}{|\Delta|} \inf _{\mathbb{F} \in \mathcal{F}}\|\partial \Delta\|_{|\mathbb{H}-\mathbb{F}|}^{2}
$$

and $C_{o s c}$ depends only on $d$.

The oscillation terms are conventional in the contemporary error analysis. Their value depend on the simplex and particular features of the function. For smooth solutions and shape-regular simplexes, the oscillation terms are much smaller than the error valiue.

\subsection{On selection of $\alpha_{k}$}

Let $\mathbb{H}\left(u_{2}\right)$ be definite. The derivation of metric $\mathfrak{M}_{\Delta}$ suggests a simple motivation for the choices (4) and (6). Since the bubble function $b_{k}$ is non-zero only on one edge, we get 


$$
\left|\left(\mathfrak{N}_{\Delta} \mathrm{e}_{k}, \mathrm{e}_{k}\right)\right|=\frac{1}{2} \alpha_{k}\left(\left|\mathbb{H}\left(b_{k}\right)\right| \mathrm{e}_{k}, \mathrm{e}_{k}\right)=4 \frac{\alpha_{k}}{\left|\gamma_{k}\right|}\left\|e_{2}\right\|_{L^{\infty}\left(\mathrm{e}_{k}\right)}
$$

where the second term is the second derivative in direction $\mathrm{e}_{k}$. For a mesh composed of $\mathfrak{M}_{\Delta \text {-equilateral simplexes, } \mathfrak{w}_{\mathrm{C}} \text { get }}$

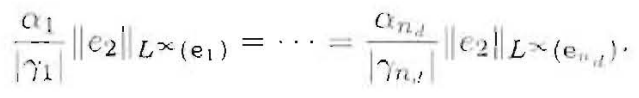

Now, in addition to the equidistribution of $\left\|e_{2}\right\|_{L^{2}}(\Delta)$, we aliso require the equidistribution of $\left\|e_{2}\right\|_{L^{x}\left(\mathrm{e}_{k}\right)}$ over all edges of each simplex $\Delta$. This requirement results in (4). We note that this choice has another advantage. In spite of local metric construction, we have approxinate equality of edge length measured in different metrics $\mathfrak{N}_{\Delta}$ coming from simplexes $\Delta$ sharing the edge. Similar arguments are used to motivate the choice (6).

\subsection{Error estimates as functions of $N_{h}$}

The error equidistribution principle suggests to build meshes that are quasiuniform in metric $\mathfrak{M}$, for the interpolation error, or in metric $\widetilde{M}$, for the gradient of the interpolation crror. Let $\Omega_{h}$ and $\widetilde{\Omega}_{h}$ be simplicial meshes with $N_{h}$ cells that balance the volume and perimeter of cells:

$$
N_{h}^{-1}|\Omega|_{\mathfrak{M}_{n}} \simeq|\Delta|_{\mathfrak{M}_{\Delta, n}} \simeq|\partial \Delta|_{\mathfrak{m}_{\Delta, n}}^{d} \quad \forall \Delta \in \Omega_{h}
$$

and

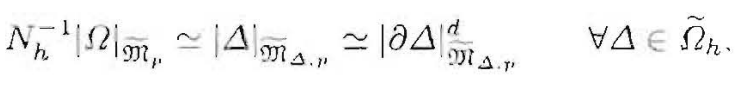

where $a \simeq b$ means that $c a \leq b \leq C a$ with constants depending only on $d$ and $p$. On such meshes, the following error estimates are held:

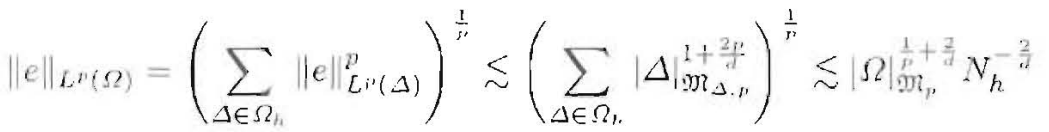

and

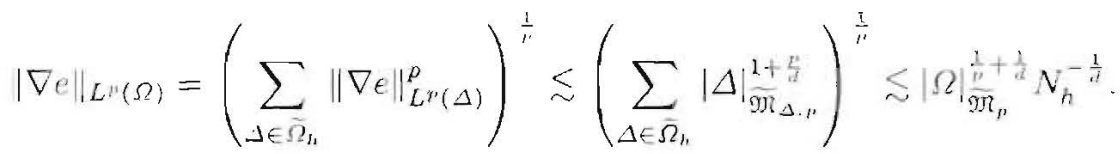

Thus, the $\mathfrak{M}_{\rho}$ (resp., $\mathfrak{M}_{p}$ )-quasi-uniform neshes provide asymptotically optimal rate for reduction of the interpolation error (resp., the gradient of the error). 


\section{Mesh adaptation algorithm}

To build a continuous metric from a piecewise constant metric, we employ the method of shifts. For every node $\mathrm{a}_{i}$ in $\Omega_{h}$, we clefine the superelement $\sigma_{i}$

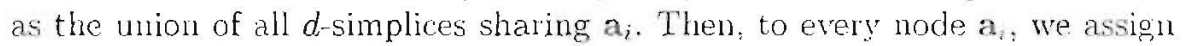
the metric with the largest determinant among all metrics associated with the superelement $\sigma_{i}$.

We use Algorithm 1 to build an adaptive mesh minimizing the $L^{\mu}$-norn of error or its gradient.

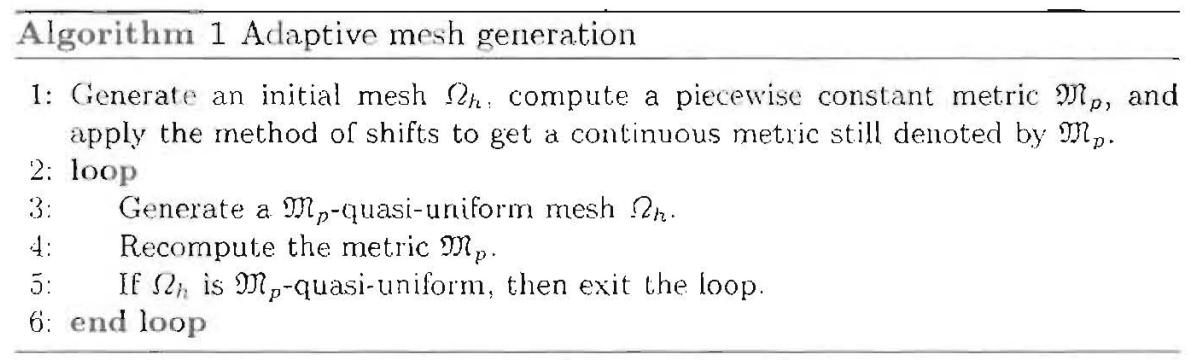

To generate a $\mathfrak{M}$-quasi-uniform mesh, we use a sequence of local mesh modifications $[2,5,15]$ that gradually increase the measure of mesh quasiuniformity. The local modifications of mesh topology include edge swapping, node relocation, insertion and deletion. These operations are implemented in package Ani2D (sourceforge.net/projects/ani2d).

\section{Numerical results}

\subsection{Interpolation problems}

In this section, we demonstrate with numerical experiments that the recovered piecewise-constant metric is sufficiently 'smooth' and reflects anisotropic features of the interpolated function. Let $\mathcal{E}^{0}$ be the set of interior mesh edges. In the two dimensional case, we define the measure of metric discontinuity as follows:

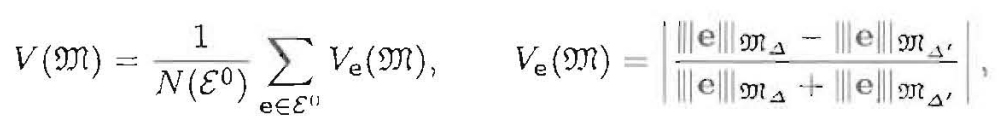

where $N\left(\mathcal{E}^{0}\right)$ is the number of interior edges and $\mathfrak{M}_{\Delta}, \mathfrak{M}_{\Delta^{\prime}}$ are two triangles with the common edge $e$. Note that $V(\mathfrak{M})$ is zero for a continuons metric. For a sequence of refined shape-regular meshes and corresponding piecewise constant metrics approximating a continuous metric: $V(\mathfrak{M})$ is converging to zero. 
In the first experiment, we calculate $V(\mathfrak{M})$ on a sequence of quasi-optimal meshes built with Algorithm 1. In the unit square $\Omega=\left[\begin{array}{ll}0,1 & 2\end{array}{ }^{2}\right.$, we consider the analytical function proposed in [9]:

$$
u(x, y)=\frac{(x-0.5)^{2}-(\sqrt{10} y+0.2)^{2}}{\left((x-0.5)^{2}+(\sqrt{10} y+0.2)^{2}\right)^{2}} .
$$

The function has an anisotropic singularity at point $(0.5,-0.2 / \sqrt{10})$ located outside the computational domain but close to its boundary. Table 1 shows that $V(\mathfrak{M})$ is roughly 0.1 , i.e. the length of edge e varies roughly $20 \%$ when measured in metrics $\mathfrak{M}_{\Delta}$ and $\mathfrak{M}_{\Delta^{\prime}}$ associated with this edge. Lack of convergencc of $V(\mathfrak{M})$ to 0 as $N_{h} \rightarrow \infty$ may be related to the fact that the mesh is only quasi-uniform in metric $\mathfrak{M}$.

The $L^{\infty}$-norm of the interpolation elror is proportional to $N_{h}^{-1}$, while the $L^{\infty}$-norm of its gradient is proportional to $N_{h}^{-0.5}$. Note that the meshes minimizing the interpolation error and its gradient are different (see Fig. 1). The figure indicates sharper features of the gradient of the error, which is the expected result.

\begin{tabular}{r|c|c||c|c} 
& \multicolumn{2}{|c||}{ Interpolation error } & \multicolumn{2}{c}{ Gradient of interpolation error } \\
\hline \multicolumn{1}{c|}{$N_{h}$} & $\|e\|_{L^{\infty}(\Omega)}$ & $V(\mathfrak{M})$ & $\|\nabla e\|_{L^{\infty}(\Omega)}$ & $V(\mathfrak{N})$ \\
\hline 1000 & $8.29 \mathrm{e}-2$ & 0.122 & $5.41 \mathrm{e}+1$ & 0.119 \\
4000 & $2.36 \mathrm{e}-2$ & 0.114 & $2.70 \mathrm{e}+1$ & 0.097 \\
16000 & $6.59 \mathrm{e}-3$ & 0.115 & $1.42 \mathrm{e}+1$ & 0.096 \\
64000 & $1.83 \mathrm{e}-3$ & 0.113 & $7.71 \mathrm{e}+0$ & 0.099 \\
\hline rate & 0.92 & & 0.48 &
\end{tabular}

Table 1. Experiment 1: convergence of the interpolation error and its gradient.

In the second experiment, we consider the Texas-shape domain inscribed in $\left[-\frac{3}{2} ; \frac{3}{2}\right]$ and shown in Fig 2 . We consider the analytical function

$$
u(x, y)=\left(x^{2} y+y^{3}\right) / 16^{3}+\tanh (2(\sin (6 y)-3 x)(\sin (6 x)-3 y))
$$

that has a spider-like distinguished feature highlighted by the mesh anisotropy. The results of numerical experiments collected in Table 2 confirm conclusions that we made in the previous experiment. We observe the first-order convergence rate for the maximum norm of the error and the half-order convergence rate for the gradient of this error. The measure of metric discontinuity $V(\mathfrak{M})$ is slowly decreasing; however, its convergence to zero is questionable. Fig. 2 shows that the meshes minimizing the interpolation error and its gradient are different, which is the expected result.

Actual numerical values of $V(\mathfrak{M})$ cause slight but yet unpleasant instabilities in the adaptive process. We found numerically that the adaptation is 

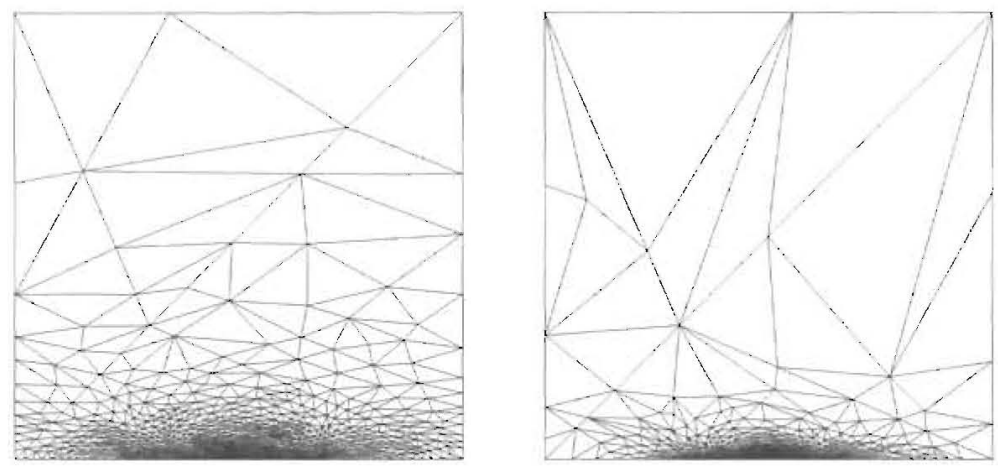

Fig. 1. Experiment 1: The adaptive meshes with roughly 2000 triangles minimizing: the maximum norm of the interpolation error (left) and its gradient (right).

\begin{tabular}{r|c|c||c|c} 
& \multicolumn{2}{|c||}{ Interpolation error } & \multicolumn{2}{c}{ Gradient of interpolation error } \\
\hline$N_{h}$ & $\|e\|_{L^{\infty}(\Omega)}$ & $V(\mathfrak{M})$ & $\|\nabla e\|_{L^{x}(\Omega)}$ & $V(\mathfrak{M})$ \\
\hline 1000 & $1.03 \mathrm{e}-1$ & 0.197 & $1.75 \mathrm{e}-0$ & 0.220 \\
4000 & $2.09 \mathrm{e}-2$ & 0.122 & $7.72 \mathrm{e}-1$ & 0.146 \\
16000 & $5.38 \mathrm{e}-3$ & 0.098 & $3.76 \mathrm{e}-1$ & 0.104 \\
64000 & $1.39 \mathrm{e}-3$ & 0.090 & $1.93 \mathrm{e}-1$ & 0.090 \\
\hline rate & 1.03 & & 0.53 &
\end{tabular}

Table 2. Experiment 2: convergence of the interpolation error and its gradient.
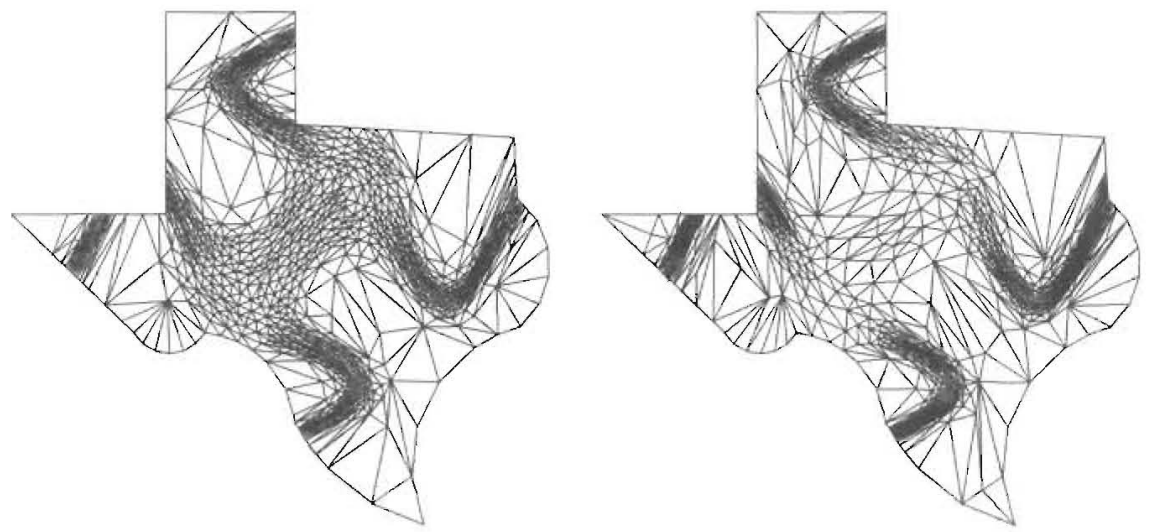

Fig. 2. Experiment 2: 'l'he adaptive meshes with roughly 2000 triangles minimizing the maximum norm of the interpolation error (left) and its gradient (right). 
more lobust for a continuous tensor metric that provides faster convergence and results in a smoother mesh. That is why we use the method of shifts to generate of a continuous metric.

\subsection{Applications to PDEs}

In this section, we apply the developed nethodology to adaptive solution of finite element problems. We consider problems with isotropic and anisotropic solutions.

\section{Hierarchical error estimates}

We describe briefyy a discretization error estimator based on enrichnent of the linear finite element space with a space of piecewise quadratic finite element functions (bubbles) associated with edges of $\Omega_{h}$ [10]. The extended finite element problem results in a system of algebraic equations:

$$
\left[\begin{array}{ll}
A_{L L} & A_{L Q} \\
A_{Q L} & A_{Q Q}
\end{array}\right]\left[\begin{array}{l}
u_{L} \\
u_{Q}
\end{array}\right]=\left[\begin{array}{l}
f_{L} \\
f_{Q}
\end{array}\right]
$$

where subscripts $L$ and $Q$ stand for linear and quadratic terms.

Let $u_{L}^{*}$ be an approximate solution of the original $P_{1}$ finite element problem $A_{L L} u_{L}^{*}=f_{L}$. We define the deviation $d_{L}=u_{L}-u_{L}$ and the discretization error $d_{Q}=u_{Q}$. They satisfy

$$
\left[\begin{array}{ll}
A_{L L} & A_{L Q} \\
A_{Q L} & A_{Q Q}
\end{array}\right]\left[\begin{array}{l}
d_{L} \\
d_{Q}
\end{array}\right]=\left[\begin{array}{l}
r_{L} \\
r_{Q}
\end{array}\right]
$$

with

$$
r_{L}=f_{L}-A_{L L} u_{L}^{*} \quad \text { and } \quad r_{Q}=f_{Q}-A_{Q L} u_{L}^{*}
$$

The exact solution of (16) is too expensive. In order to estimate the discretization error $d_{Q}$, equation (16) is replaced with a simpler equation

$$
\left[\begin{array}{cc}
A_{L L} & 0 \\
0 & A_{Q Q}
\end{array}\right]\left[\begin{array}{c}
\tilde{d}_{L} \\
\tilde{d}_{Q}
\end{array}\right]=\left[\begin{array}{c}
r_{L} \\
r_{Q}
\end{array}\right] .
$$

Using a local finite element analysis, one can show that the diagonal matrix in (17) is spectrally equivalent to the matrix in (16). Therefore, the energy norm of the discretization error $d_{Q}$ can be estimated using the energy norm of $\tilde{d}_{Q}$. The matrix $A_{Q Q}$ is well-conditioned for shape-regular meshes; therefore. the vector $\tilde{d}_{Q}$ can be efficiently calculated with a simple conjugate gradient method.

The entry of vector $\tilde{d}_{Q}$ associated with an edge $e_{k}$ of a simplex $\Delta$ plays the role of interpolation error $\gamma_{k}$ in formula (1). Thus, we can use the above methodology to generate quasi-optimal meshes. 
Problem with a point singularity

Let $\Omega$ be a unit disk with a radial cut. We consider the classical crack problem with the exact solution

$$
u(r, \theta)=r^{1 / 4} \sin (\theta / 4),
$$

where $(r . \theta)$ are polar coordinates, $r>0$ and $\theta \in[0,2 \pi)$. The crack line $S$ is defined by points $(r, 0)$. We consider the following boundary value problen:

$$
\begin{array}{rlrl}
\Delta u & =0 & & \text { in } \quad \Omega \backslash S, \\
u=\sin \frac{\theta}{4} & & \text { on } \quad \partial \Omega \backslash S, \\
u=0 & \text { on } \quad S^{i}, \quad \frac{\partial u}{\partial n}=0 \quad \text { on } S^{-},
\end{array}
$$

where $S^{+}$and $S^{-}$denote the crack line when it is approached from regions $\theta \rightarrow+0$ and $\theta \rightarrow 2 \pi$, respectively.

Table 3 demonstrates the half-order convergence of the gradient of the discretization error. A similar convergence is observed for the gradient of a finite element function $\tilde{d}_{h}$ corresponding to vector $\tilde{d}_{Q}$, which confirms the theory of hierarchical error estimates on shape-regular meshes. The difference in error values indicates that the constant of spectral equivalence of energy norms of $d_{Q}$ and $\tilde{d}_{Q}$ is approximately 6 .

This theory does not guarantee a similar connection between $L^{2}$-norms of these error's, which is also clear from the second and third columns in Table 3. For this norm, we have to use the finite element function $d_{h}$ corresponding to $d_{Q}$; however, calculation of this function is rather expensive.

\begin{tabular}{r|c|c||c|c} 
& \multicolumn{2}{|c||}{ Discretization error } & \multicolumn{3}{c}{ Gradient of discretization error } \\
\hline$N_{h}$ & $\left\|\tilde{d}_{h}\right\|_{L^{2}(\Omega)}$ & $\|e\|_{L^{2}(\Omega)}$ & $\left\|\nabla d_{h}\right\|_{L^{2}(\Omega)}$ & $\|\nabla e\|_{L^{2}(\Omega)}$ \\
\hline 1000 & $1.10 \mathrm{e}-3$ & $7.44 \mathrm{e}-3$ & $1.94 \mathrm{e}-2$ & $1.17 \mathrm{e}-1$ \\
4000 & $2.84 \mathrm{e}-4$ & $3.10 \mathrm{e}-3$ & $1.01 \mathrm{e}-2$ & $6.08 \mathrm{e}-2$ \\
16000 & $7.04 \mathrm{e}-5$ & $1.30 \mathrm{e}-3$ & $5.35 \mathrm{e}-3$ & $2.96 \mathrm{e}-2$ \\
64000 & $1.76 \mathrm{e}-5$ & $9.48 \mathrm{e}-4$ & $2.62 \mathrm{e}-3$ & $1.59 \mathrm{e}-2$ \\
\hline rate & 1.00 & 0.51 & 0.48 & 0.48
\end{tabular}

Table 3. Experiment 3: convergence of the discretization error and its gradient.

\section{Problem with anisotropic singularities}

Let $\Omega$ be the unit square $\Omega=(0,1)^{2}$. We consider the following boundary value problem: 

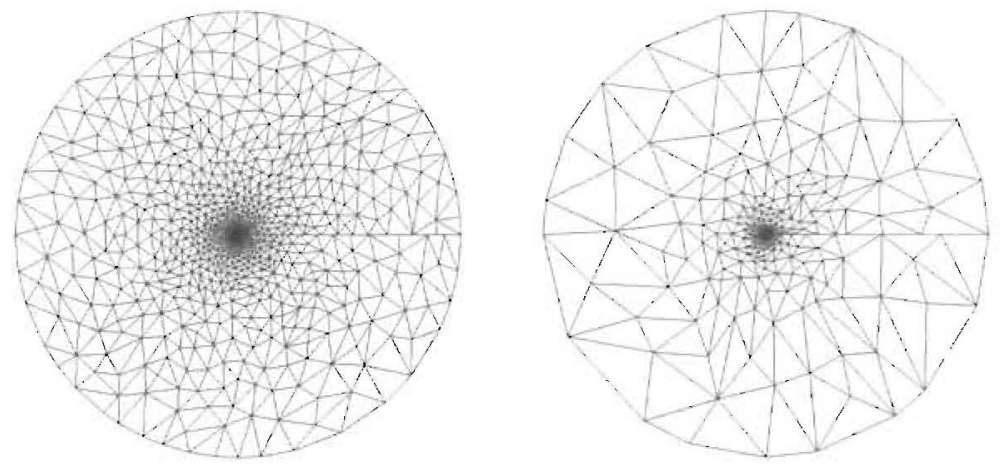

Fig. 3. Experinent 3: The adaptive meshes with roughly 2000 triangles minimizing the $L^{2}$-norm of the hierarchical error estimator (left) and its gradient (right).

$$
\begin{aligned}
-\operatorname{div}(\mathbb{K} \operatorname{grad} u) & =1 & & \text { in } \Omega, \\
u & =0 & & \text { on } \partial \Omega,
\end{aligned}
$$

where

$$
\mathbb{K}(x, y)=\mathbb{R}_{\theta}^{T}\left[\begin{array}{cc}
1 & 0 \\
0 & 10^{3}
\end{array}\right] \mathbb{R}_{\theta}, \quad \theta=250(x+y),
$$

and $\mathbb{R}_{\theta}$ is the rotation matrix by angle $\theta$. The analytical solution is unknown and the discretization error cannot be computed. However, some features of the solution can be extracted from the mesh structurc shown in Fig. 4. Table 4 shows that the gradient of hierarchical edge-based a posteriori error estimator, $\left\|\nabla \tilde{d}_{h}\right\|_{L^{2}(\Omega)}$, correlates with $\left\|\nabla d_{h}\right\|_{L^{2}(\Omega)}$ even on anisotropic meshes. Similarly to the previous experiment, the $L^{2}$-norms of these estimators exhibit different behavior.

\begin{tabular}{r|c|c||c|c} 
& \multicolumn{2}{|c||}{ Discretization error } & \multicolumn{2}{c}{ Gradient of discretization error } \\
\hline$N_{h}$ & $\left\|\tilde{d}_{h}\right\|_{L^{2}(\Omega)}$ & $\left\|d_{h_{h}}\right\|_{L^{2}(\Omega)}$ & $\left\|\nabla \tilde{d}_{h}\right\|_{L^{2}(\Omega)}$ & $\left\|\nabla d_{h}\right\|_{L^{2}(\Omega)}$ \\
\hline 1000 & $4.98 \mathrm{e}-6$ & $5.55 \mathrm{e}-3$ & $1.88 \mathrm{e}-4$ & $6.52 \mathrm{e}-4$ \\
4000 & $1.48 \mathrm{e}-6$ & $3.02 \mathrm{e}-3$ & $1.16 \mathrm{e}-4$ & $3.52 \mathrm{e}-4$ \\
16000 & $3.88 \mathrm{e}-7$ & $1.82 \mathrm{e}-3$ & $5.94 \mathrm{e}-5$ & $1.67 \mathrm{e}-4$ \\
64000 & $9.87 \mathrm{e}-8$ & $1.03 \mathrm{e}-3$ & $2.94 \mathrm{e}-5$ & $9.07 \mathrm{e}-5$ \\
\hline rate & 0.95 & 0.40 & 0.45 & 0.48
\end{tabular}

Table 4. Experiment 4: convergence of the hierarchical error estimators $\tilde{d}_{h}, d_{h}$, and their gradients. 

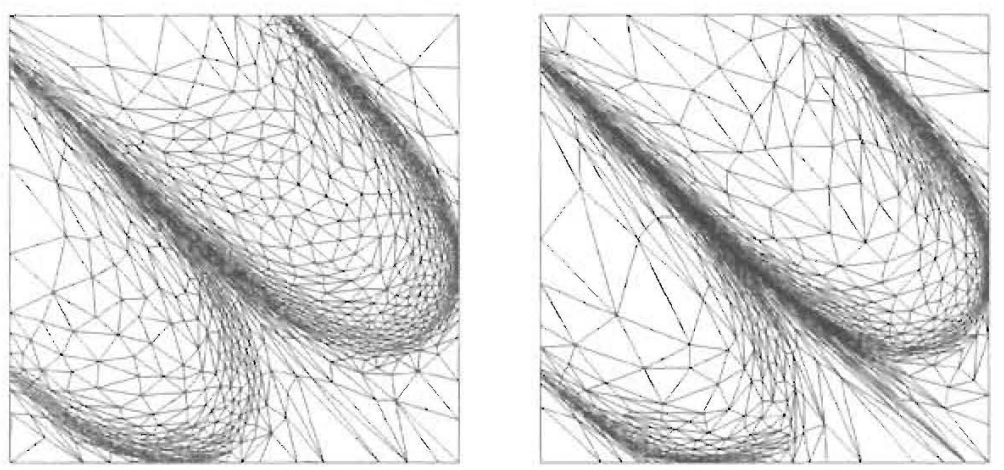

Fig. 4. Experiment 4: The adaptive meshes with roughly 2000 triangles minimizing the $L^{2}$-norm of the hierarchical error estimator $\tilde{d}_{h}$ (lelt) and its gradient (right).

\section{Conclusion}

We presented a new technology for generating meshes minimizing the interpolation and discretization errors or their gradients. The cornerstone of this methodology is construction of a space metric from edge-based error estimates. For the interpolation error, these estimates were computed explicitly. For the discretizations error, we used the hierarchical error estiniators based on enrichment of the linear finite element space with quadratic bubble functions associated with mesh edges. We proved and verified with numerical experiments, that for a mesh with $N_{h}$ triangles, the error is proportional to $N_{h}^{-1}$ and the gradient of this error is proportional to $N_{h}^{-1 / 2}$, which are optimal asymptotics.

\section{References}

1. Agouzal A, Vassilevski $Y$ (2008) Minimization of gradient errors of piecenise linear interpolation on simplicial meshes. Submitted to SIAM J. Numer. Anal.

2. Agouzal A, Lipnikov K, Vassilevski Y (1999) Adaptive generation of quasioptimal tetrahedral meshes. East-West J. Numer. Math. 7, 223-244.

3. Agouzal A, Lipnikov K, Vassilevski Y (2007) Generation of quasi-optimal meshes based on a posteriori error estimates. Proceedings of 16 th International Meshing Roundtable (M.Brewer, D.Marcum; Editors), Springer: 139-148.

4. Agouzal A, Lipnikov K, Vassilevski Y (2009) Hessian-frete netric-based mesh adiptation via geometry of interpolation error Corre. Math Math. Phys. 49(11), to appear.

5. Buscaglia GC, Dari EA (1997) Anisotropic mesh optimization and its application in adaptivity. Inter. J. Numer. Weth. Engrg. 40, 4119-4136.

6. Chen L. Sun P. Xu J (2007) Optimal anisotropic meshes for minimizing interpolation errors in $L^{p}$-norm. Mathematics of Computation 76, 179-204. 
7. Ciarlet P. Wagschal C (1971) Multipoint 'Laylor formulas and applications to the finite element method, Numer. Math.. 17, 84-100.

8. Ciarlet P, The finile element method for elliptic problems, North-Holland, 1978.

9. D'Azevedo E (1991) Optimal triangular mesh generation by coordinate transformation. SIAM J. Sci. Stat. Comput. 12, 755-786.

10. Deuflhard P., Leinen P., Y'serentant H. (1989) Concepts of an adaptive hierarchical finite element code, IMPAC' 1, 3-35.

11. Frey PJ, Alauzet E (2005) Anisotropic mesh adaptation for CFD computations. Comput. Meth. Appl. Mech. Eng. 194, 5068-5082.

12. Huang $W$ (2005) Metric tensors for anisotropic niesh generation. J. Comp. Physics. 204. 633-665.

13. Lipnikov K, Vassilevski Y (2003) Parallel adaptive solution of 3D) bomulary value problems by Hessian recovery. Comput. Meth. Appl. Mech. Eng. 192. $1495-1513$.

1:1. 'Lichomirov V.M. (1960) The widths of sets in functional spaces and theory of optimal approximations, Uspehi Matem. Nauk, 15(3), 81-120.

15. Vassilevski Y, Lipnikov K (1999) Adaptive algorithm for generation of quasioptimal meshes. Comp. Math. Math. Phys. 39, 1532-1551.

16. Vassilevski Y, Agouzal A (2005) An unified asymptotic analysis of interpolation errors for optimal meshes. Doklady Mathematics 72, 879-882.

1.7. Zhu JZ, Zienkiewicz OC (1990) Superconvergence recovery tochnique and a posteriori error estimators. Inter. J. Numer. Meth. Engrg. 30, 1321-1339. 